\title{
A Cross-Layer Approach to End-to-End Routing and SINR-based Scheduling in Multi-hop Wireless Networks
}

\author{
Sastry Kompella $^{\dagger} \quad$ Jeffrey E. Wieselthier ${ }^{\dagger}$ Anthony Ephremides ${ }^{\ddagger} \quad$ Hanif D. Sherali ${ }^{\triangleright}$ \\ $\dagger$ Information Technology Division, Naval Research Laboratory, Washington, DC \\ $\ddagger$ Electrical and Computer Eng. Dept., University of Maryland, College Park, MD 20742 \\ $\diamond$ Grado Department of Industrial and Systems Engineering, Virginia Tech, Blacksburg, VA 24061
}

\begin{abstract}
In this paper we study the problem of jointly controlling routing and transmission scheduling in spatial TDMA (STDMA)-based multihop wireless networks, with the goal of determining the minimum-length schedule that satisfies a given end-to-end traffic demand. We present a cross-layer formulation of the problem that incorporates multi-path routing at the network layer, while concurrently generating matchings to address the media-access control problem. Each such matching consists of a set of links that can simultaneously be active, without violating the specified signal-to-interference-and-noise ratio (SINR) requirement. We formulate our problem as one of mixed integer-linear programming, and we show that our column generation approach converges to a globally optimal solution. After considering a problem formulation that is restricted to the use of the same $R F$ transmission power by all nodes, we extend our model to incorporate power adaptation, and demonstrate the resulting improvement in performance. We also discuss the insights that can be gained about the influence of power control, and spatial reuse on network performance.
\end{abstract}

\section{INTRODUCTION}

Resource optimization in multi-hop wireless networks has recently received considerable attention ( [1], [4]-[6]). Multi-hop wireless networks are of interest not only in military command and control systems, but also in civilian applications such as disaster relief, urban wireless connectivity, etc. It is widely accepted that contention-based medium access schemes are not well suited for providing QoS guarantees, such as delay bounds, in multi-hop wireless networks. It is also understood that scheduling, as an access control method, can avoid collisions and retransmissions of contentionbased methods. Whereas scheduling methods such as time division multiple access (TDMA) schemes can guarantee such delay bounds, their efficiency can be further improved both in terms of delay guarantees as well as achieving higher capacities by allowing the TDMA time-slots to be shared by simultaneous transmissions that are geographically separated, such that the resulting interference is minimized. This scheme is appropriately termed as Spatial-TDMA (STDMA) [4].

In the case of STDMA-based medium access control, Bjorklund et al. [1] showed that even the most basic planning problems of STDMA node and link assignment are NP-hard. The objective of the optimization problem in [1] was to minimize the length of the STDMA frame such that either all nodes or all network links are assigned to at least one time slot. In [3], we presented the problem of determining the minimumlength schedule that satisfies traffic demands for a set of chosen links. This set of links could constitute valid paths between source-destination pairs, in which case we can imagine that the paths are chosen $a$ priori. However, in wireless networks, any such path selection without explicit physical layer considerations is clearly sub-optimal. Therefore, in this paper, we study the problem of resource allocation and STDMAbased link scheduling optimization in multi-hop wireless networks, by jointly determining routing and the minimum-length schedule in order to satisfy an end-toend traffic demand. Our efforts in this paper are closely related to the works discussed above and our main contribution is to extend the previous approaches by introducing routing between source-destination pairs, i.e., the objective of the minimum length scheduling problem is to compute the shortest schedule that can satisfy the end-to-end traffic demands for a set of source-destination pairs rather than satisfying a given traffic demands for each link (which presupposes the use of an arbitrarily chosen set of routes that is mostlikely non-optimal). Furthermore, the problem formulation also provides the means to incorporate various power and rate adaptation schemes, while seamlessly integrating the SINR constraints for generating active sets of links (or matchings). Specifically, for a given 
network configuration and a given end-to-end traffic demands, we formulate the minimum-length scheduling problem with routing [MLSPR] as a linear programming (LP) problem, under the assumption that all the feasible matchings are already enumerated. However, since the number of such enumerations could be very large, this problem formulation is efficient only for relatively small networks. Therefore, we present an efficient solution procedure based on column generation that selectively enumerates only those matchings that contribute towards determining an optimal solution.

The rest of this paper is organized as follows. In Section II, we formulate the minimum-length scheduling problem with routing. We then propose a column generation-based solution procedure for jointly determining the routes and the minimum length schedule in Section III, and present numerical results in Section IV. In Section V we provide conclusions for this paper.

\section{Problem Formulation}

\section{A. Network and Communication Model}

We model a multi-hop wireless network as a set of stationary nodes $\mathcal{N}$. Each node acts as a transmitter and receiver, and also relays data to neighboring nodes. A set of (directed) links $\mathcal{E}$ constitutes the network topology, and link $\{i, j\} \in \mathcal{E}$ means that node $i$ can communicate directly with node $j$, i.e., the corresponding signal-to-noise ratio (SNR) in the absence of any other interference source exceeds a specific threshold. That is, the graph representation of the wireless network is based on whether a node can reach another node when transmitting in isolation for a given power, noise level and channel gain.

Let $P_{i}$ be the transmission power for node $i, G_{i j}$ the channel gain between nodes $i$ and $j$, and $\eta_{j}$ be the thermal noise at receiver $j$. In the physical model, the SINR at receiver $j$ due to transmission from node $i$ in the presence of other transmissions is given by:

$$
S I N R_{i j}=\frac{P_{i} G_{i j}}{\eta_{j}+\sum_{k \neq i, j} P_{k} G_{k j}} .
$$

The channel gain is calculated by the widely used farfield model $G_{i j}=d_{i j}^{-\alpha}$, where $d_{i j}$ is the distance between nodes $i$ and $j$, and $\alpha$ (which typically takes on a value between 2 and 4) is the path loss index.

The capacity of the wireless channel associated with a link $(i, j)$ is a function of SINR on the channel. We assume that data is coded separately for each link and that the receivers consider unintended receptions as noise (i.e., we do not consider the possibility of multi-user detection) In this case, each link $(i, j)$ can be viewed as a single-user Gaussian channel, and the Shannon capacity over a frequency band $W$ is given by $c_{i j}=W \log _{2}\left(1+S I N R_{i j}\right)$. In practice, however, it is understood that most communication schemes will achieve lower rates.

\section{B. Scheduling}

Definition 1: Given a set of links $M \subseteq \mathcal{E}$, all links in $M$ can be activated concurrently if such simultaneous activation does not violate the minimum SINR required for communication, i.e., the SINR threshold is satisfied across all links in $M$. A set $M$ satisfying this condition is called a "feasible matching", or simply, a matching.

Definition 2: A schedule is defined as an indexed collection $\mathcal{S}=\left(M^{s}, \lambda^{s}, s \in S\right)$, where the index set $S$ is an arbitrarily large but finite, $M^{s}$ is a matching for each $s$, and $\lambda^{s} \geq 0$ (which is a continuous variable) is the duration that the links in $M^{s}$ are simultaneously active in periodically recurring frames.

The schedule length $\tau$ of the schedule $\mathcal{S}$ is given by $\tau=\sum_{s} \lambda^{s}$. This schedule $\mathcal{S}$ can be implemented in an STDMA framework, in which transmission resources are divided into time-slots, and a link may be active in one or several time-slots based on how many matchings contain this link.

\section{Routing}

Consider $L$ concurrent sessions, each of which corresponds to a source-destination pair in the network. The traffic demand for each session $l, 1 \leq l \leq L$, is given by $R_{l}$ which is to be transmitted from the source node $s_{l}$ to destination node $d_{l}$, along a set of links that constitute a route for each session $l$. In this paper, the traffic demand $R_{l}$ is expressed in terms of bits per frame, where the frame length is not specified apriori. An alternative formulation in which $R_{l}$ is expressed in terms of bits per second, is discussed in [3].

In order to relay this traffic demand for each session, we take advantage of the availability of multiple paths between source-destination pairs, and allow the source to split the data into multiple sub-flows if necessary. We denote the data rate associated with the $l$-th session on link $(i, j)$ by $r_{i j}^{l}$.

Then, for a node $i$, which is the source node of session $l$ (i.e., $i=s_{l}$ ), the following condition holds: 


$$
\sum_{j \in \mathcal{N}(i)} r_{i j}^{l}=R_{l}
$$

where $\mathcal{N}(i)$ denotes the set of all nodes having links that originate at node $i$, i.e., the one-hop neighbors of node $i$.

If node $i$ is an intermediate relay node for the $l$-th session (meaning, $i \neq s_{l}$ and $i \neq d_{l}$ ), then, the flow balance equations can be written as follows:

$$
\sum_{j \in \mathcal{N}(i)} r_{i j}^{l}-\sum_{j: i \in \mathcal{N}(j)} r_{j i}^{l}=0 .
$$

Finally, if node $i$ is the destination node, $i=d_{l}$, we have

$$
-\sum_{j: i \in \mathcal{N}(j)} r_{j i}^{l}=-R_{l} .
$$

Constraints (2)-(4) for all $l, 1 \leq l \leq L$ can be concisely written as follows:

$$
\mathbf{A r}=\mathbf{R}
$$

where $A \in Z^{|\mathcal{N}| L \times|\mathcal{E}| L}$ is an integer matrix whose entries are either $1,-1$ or 0 , and $\mathbf{R}$ is the rate vector with zero-valued entries for intermediate relay nodes. The vector $\mathbf{r}$ represent the set of data rate variables $\left\{r_{i j}^{l}\right\}$.

\section{Minimum-Length Scheduling Problem with Routing}

As defined earlier, a schedule $\mathcal{S}=\left(M^{s}, \lambda^{s}, s \in S\right)$ is a collection of matchings $M^{s}$ and corresponding nonnegative values $\lambda^{s}$, such that each of the matchings is feasible, and the end-to-end demands of all sessions are satisfied. Our objective is to minimize the schedule length $\tau=\sum_{s} \lambda^{s}$. A source-destination pair associated with a session $l$ has $R_{l}$ bits of information to be transferred from source to destination in each STDMA frame. This information can be split and sent across multiple routes, and the traffic demand on link $\{i, j\}$ resulting from all such sessions $1 \leq l \leq L$ is given by $r_{i j}=\sum_{1 \leq l \leq L} r_{i j}^{l}$, which is the amount of information that needs to be transmitted in every frame, for each link $\{i, j\}$. This information transfer can be completed in a time interval or frame of length $\tau$ seconds as follows. Each frame is partitioned into subintervals or slots indexed by $s \in S$, such that in each slot, the corresponding matching $M^{s}$ is active for a duration of $\lambda^{s}$ seconds. Thus a given link $\{i, j\}$ is active during all the slots that correspond to $s$, where $\{i, j\} \in M^{s}$. Given the set $\mathcal{M}$ of all possible feasible matchings for a network, where $M^{s} \in \mathcal{M}$, the Minimum-Length Scheduling Problem with Routing [MLSPR] can be formulated as follows.

\section{[MLSPR]:}

$$
\begin{array}{ll}
\text { Minimize: } \tau=\sum_{1 \leq s \leq|\mathcal{M}|} \lambda^{s} & \\
\text { subject to: } & \mathbf{A r}=\mathbf{R} \\
\sum_{1 \leq s \leq|\mathcal{M}|} c_{i j}^{s} \lambda^{s} \geq \sum_{1 \leq l \leq L} r_{i j}^{l}, \quad \forall\{i, j\} \in \mathcal{E} \\
r_{i j}^{l} \geq 0, \quad \forall\{i, j\} \in \mathcal{E}, 1 \leq l \leq L \\
\lambda^{s} \geq 0, \quad \forall s=1, \ldots,|\mathcal{M}|
\end{array}
$$

Another possible (but essentially equivalent) interpretation for a schedule $\mathcal{S}=\left(M^{s}, \lambda^{s}, s \in S\right)$, its length $\tau$, and the link demands $r_{i j}=\sum_{l} r_{i j}^{l}$ that are derived from end-to-end demands, is presented in [3].

\section{Minimum-Length Schedule Computation}

It can be observed that [MLSPR] is a linear programming problem with a bounded optimal solution. Even though [MLSPR] has a very simple constraint structure, the complexity of the problem lies in the computation of the set of all feasible matchings $\mathcal{M}$. The number of such feasible matchings that would have to be enumerated in order to compute an optimum may be as large as $2^{|\mathcal{E}|}$. Therefore, [MLSPR] is not computationally efficient and should be solved in such a way that does not require the explicit enumeration of all matchings (each of which constitute columns in the linear program), for this would take at least $\mathcal{O}\left(2^{|\mathcal{E}|}\right)$ time. Since most columns will not be used in an optimal solution anyway, it is useful to develop techniques that can reduce the size of the search space. A column generation approach [10] is a viable alternative in such a situation. In this approach, the original problem is decomposed into a master problem and a subproblem. The strategy of this decomposition procedure is to operate iteratively on two separate, but easier-to-solve, problems. The master problem passes down a new set of cost coefficients to the subproblem, and receives a new column (i.e., a matching) based on these cost coefficients from the subproblem. 


\section{A. Master Problem}

The master problem is a restriction of the original problem [MLSPR], which uses only a subset of available columns indexed by $s \in \mathcal{S} \subseteq\{1, \ldots,|\mathcal{M}|\}$. Since this formulation optimizes over a subset of feasible matchings, an optimal solution to the master problem provides an upper bound $U B$ for [MLSPR]. The key idea of the column generation algorithm is to sequentially improve the upper bound by identifying new matchings and adding them to the master problem.

$$
\text { Minimize: } \tau=\sum_{s \in \mathcal{S}} \lambda^{s}
$$

subject to:

$$
\begin{aligned}
& \mathbf{A r}=\mathbf{R} \\
& \sum_{s \in \mathcal{S}} c_{i j}^{s} \lambda^{s} \geq \sum_{1 \leq l \leq L} r_{i j}^{l}, \quad \forall\{i, j\} \in \mathcal{E} \\
& r_{i j}^{l} \geq 0, \quad \forall\{i, j\} \in \mathcal{E}, 1 \leq l \leq L \\
& \lambda^{s} \geq 0, \quad \forall s \in \mathcal{S} .
\end{aligned}
$$

\section{B. Feasible Matchings}

During every iteration, when the master problem is solved, we need to determine whether the current solution is optimal. If not, we identify a new matching that can improve the current solution, i.e., ascertain new columns to enter into the basis (recall that each matching constitutes one column in the master problem). This can be achieved by examining whether any new matching that is not currently in the master problemhas a "negative reduced cost". Denoting the dual variables corresponding to constraint set (10) by $\left(\bar{\omega}_{i j}\right)$, the reduced cost $\bar{z}_{s}$ for a column $s$ can be expressed as:

$$
\bar{z}_{s}=1-\sum_{\{i, j\} \in \mathcal{E}} \bar{\omega}_{i j} c_{i j}^{s} .
$$

Note that $c_{i j}^{s} \equiv 0$ if the corresponding link $\{i, j\}$ is inactive. Hence, in order to find a new column having the "most negative reduced cost", we solve a subproblem with the objective given by:

$$
\operatorname{Min}_{s \in \mathcal{M}} \bar{z}_{s}=1-\operatorname{Max}_{s \in \mathcal{M}} \sum_{\{i, j\} \in \mathcal{E}} \bar{\omega}_{i j} c_{i j}^{s} .
$$

The nature of this scheduling subproblem depends on the power/rate adaptation schemes that are employed as well as any restrictions on the transmission between nodes. Based on the optimal solution to the scheduling subproblem, a non-negative reduced cost implies that the current solution to the master problem is indeed an optimal solution to [MLSPR]. Otherwise, the matching that is identified by the subproblem, which must then belong to $\mathcal{M} \backslash \mathcal{S}$, is added to the current schedule $\mathcal{S}$, and the master problem is re-optimized.

In the rest of this section, we identify different ways of generating feasible matchings and also provide the corresponding subproblem formulations.

1) Fixed Transmit Power: In this scheme, the source nodes of all active links in the matching use their maximum RF transmission power $P_{\max }$, with the condition that the SINR of all the active links in the matching exceeds a fixed threshold $\gamma$. Associated with $\gamma$ is a transmission rate $c_{i j}$ at which each active link $\{i, j\}$ would be allowed to transmit. Apart from the SINR threshold $\gamma$, the transmission rate $c_{i j}$ also depends on other factors such as the specified bit error rate constraint. However, for the sake of simplicity, we assume in this paper that the transmission rate $c_{i j}$ is computed based on the Shannon capacity formula.

Given a set of dual variables $\left(\omega_{i j}\right)$ (obtained from the master problem), a new matching can be generated by solving the corresponding subproblem shown below. The formulation of the subproblem is explained in greater detail in [3].

$$
\begin{aligned}
& \text { Maximize: } \sum_{\{i, j\} \in \mathcal{E}} \omega_{i j} c_{i j} x_{i j} \\
& \text { subject to: } \\
& \left(\eta_{j}+\sum_{k \neq i, j} G_{k j} P_{\max }-\gamma^{-1} G_{i j} P_{\max }\right) x_{i j} \\
& +\sum_{k, m \neq i, j} G_{k j} P_{\max } x_{k m} \leq \sum_{k \neq i, j} G_{k j} P_{\max }, \forall\{i, j\} \in \mathcal{E} \\
& \sum_{j:\{i, j\} \in \mathcal{E}} x_{i j}+\sum_{j:\{j, i\} \in \mathcal{E}} x_{j i} \leq 1, \quad \forall i \in \mathcal{N} \\
& x_{i j} \in\{0,1\}, \quad \forall\{i, j\} \in \mathcal{E} .
\end{aligned}
$$

Note that, in this scheme, all the links utilize the same rate of transmission, even if the link exceeds its SINR threshold. This model of communication has been commonly used in the literature because of its simplicity (see [1] and references therein). However, by fixing the powers to the maximum value $P_{\max }$, this scheme is inefficient for two reasons. First, by fixing the transmit powers, one does not gain any improvement in the transmission rate, even if the SINR on some links exceeds the threshold $\gamma$ by a wide margin, resulting in 
TABLE I

DATA SESSIONS AND TRAFFIC DEMAND FOR THE 15-NODE NETWORK

\begin{tabular}{|c|c|}
\hline Source $\rightarrow$ Dest. & $\begin{array}{c}\text { Demand } \\
(\text { Mbps/Frame })\end{array}$ \\
\hline $1 \rightarrow 10$ & 5 \\
$3 \rightarrow 12$ & 5 \\
$8 \rightarrow 13$ & 5 \\
\hline
\end{tabular}

wastage of energy. Second, the lack of power adaptation could lead to a higher interference, resulting in a fewer number of links that can be simultaneously activated.

2) Variable Transmit Power: In this case, the source nodes of all active links in the matching can transmit up to their maximum power $P_{\max }$ while satisfying the SINR constraints i.e., the SINR of all the active links exceeds a fixed threshold $\gamma$. As in the fixed power case, the formulation for the case of variable transmit power is explained in greater detail in [3]. The resulting subproblem is as follows:

Maximize: $\sum_{\{i, j\} \in \mathcal{E}} \omega_{i j} c_{i j} x_{i j}$

subject to:

$$
\begin{aligned}
& \gamma\left(\eta_{j}+\sum_{k \neq i, j} G_{k j} P_{\max }\right) x_{i j}+\gamma \sum_{k \neq i, j} G_{k j} P_{k}-G_{i j} P_{i} \leq \\
& \gamma \sum_{k \neq i, j} G_{k j} P_{\max }, \quad \forall\{i, j\} \in \mathcal{E} \\
& \sum_{j:\{i, j\} \in \mathcal{E}} x_{i j}+\sum_{j:\{j, i\} \in \mathcal{E}} x_{j i} \leq 1, \quad \forall i \in \mathcal{N} \\
& x_{i j} \in\{0,1\}, \quad \forall\{i, j\} \in \mathcal{E} \\
& 0 \leq P_{i} \leq P_{\max }, \quad \forall i \in \mathcal{N} .
\end{aligned}
$$

\section{NUMERICAL RESULTS}

In this section, we present numerical results that offer further insights into the joint routing and transmission scheduling problem [MLSPR]. We consider a randomly generated network of 15 nodes deployed over a $50 \mathrm{~m}$ $\mathrm{x} 50 \mathrm{~m}$ area. As described earlier, the topology is determined by whether a node can reach any other node when transmitting in isolation at its peak power $P_{\max }$. Here we set $P_{\max }=0.1$ Watts. The 15 -node network is constructed using a radio link model that has a bandwidth $W=1 \mathrm{MHz}$. The path loss index is chosen as $\alpha=2$, and the thermal noise at the receiver is given by $\eta=10^{-5}$ Watts. The SINR threshold is chosen as $\gamma=2$.

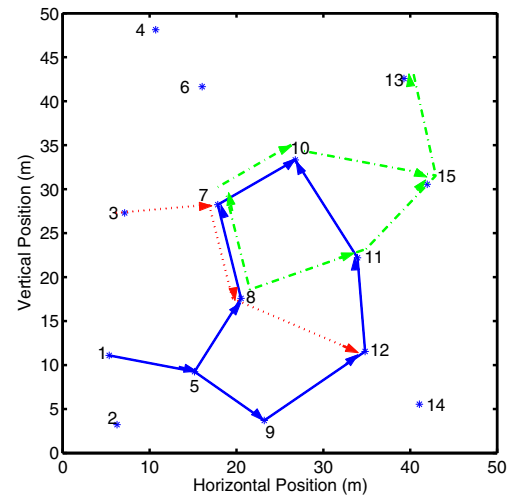

Fig. 1. Optimal paths for a 15-node network using fixed power and source-destination pairs described in Table I.

The proposed solution procedure is implemented in C on a Pentium 4 desktop with 1GB of RAM, and the LINDO API 4.0 is used as the optimization tool for solving the master problem as well as the respective mixed integer subproblems.

\section{A. Identifying Optimal Routes}

In order to evaluate the performance of the column generation based solution procedure in computing the optimal paths for each source destination pair, we utilize the 15-node network with three concurrent data sessions. The source and destination node details for the data sessions are shown in Table I, along with their end-to-end traffic demands. The set of final optimal routes that are identified by the algorithm are shown in Figures 1 and 2.

Figure 1 shows the optimal paths when the transmit power is fixed between nodes as discussed in Section III-B.1. In the ensuing algorithm, the corresponding subproblem is solved in order to generate a new matching in every iteration, and the active nodes in each such newly generated matching transmit at the maximum power $P_{\max }$. The resulting schedule length is presented in Table II. Figure 2 shows the optimal routes in the variable transmit power case. As discussed earlier, our problem formulation allows for data transmission over multiple paths between the source and destination. This is evident, for example in Figure 1, where the data bound for node 10 from source node 1 is split at node 5 to be transmitted over two sub-paths.

\section{B. Comparison of Routing Strategies}

For the 15-node network in Figures 1 and 2, we now consider the impact of routing on the computation of the 
TABLE II

Schedule Length (SEC.) FOR Different Routing Strategies

\begin{tabular}{|c|c|c|}
\hline Routing Strategy & Fixed Power & Variable Power \\
\hline Joint Routing and Scheduling [MLSPR] & 15.77 & 14.66 \\
\hline Minimum-energy & 22.50 & 21.10 \\
\hline Minimum-Hop & 21.95 & 21.95 \\
\hline
\end{tabular}

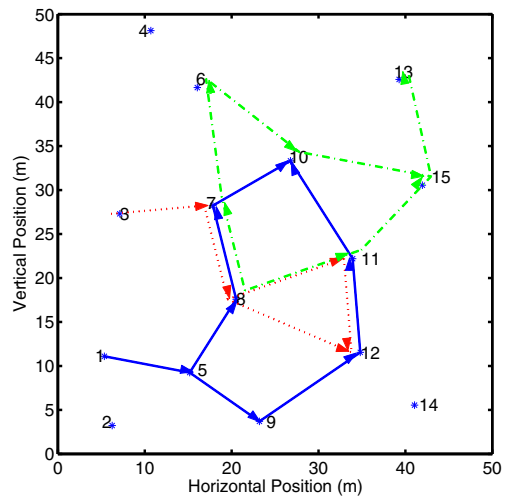

Fig. 2. Optimal paths for a 15-node network using variable power and source-destination pairs described in Table I.

minimum-length schedule. Similar to our investigation in [3], for comparison we consider the following two routing approaches, namely, minimum-energy routing and minimum-hop routing. Under the minimum-energy routing, the energy cost is defined as $d_{i j}^{\alpha}$ for any given link $\{i, j\}$. Here again, we consider three data sessions and the corresponding traffic demands shown in Table I. The results are shown in Table II. Clearly the joint routing and scheduling strategy outperforms both minimum-hop and minimum-energy routing in terms of the schedule length. The reason is that, even though minimum-energy routing and minimum-hop routing uses a fewer number of hops, the combined routing and scheduling algorithm finds paths that would allow for generating matchings with higher spatial reuse factor in order to minimize the schedule length. Here, spatial reuse factor is defined as the average number of links that can be activated simultaneously in a matching. Spatial reuse improves in the presence of transmit power control of nodes, resulting in a further improvement in schedule length when compared with the fixed transmit power scheme.

\section{CONCLUSION}

In this paper, we presented the problem of jointly determining routing and the minimum-length schedule problem in STDMA-based multi-hop wireless networks. This cross-layer problem has been formulated by considering routing at the network layer, scheduling at the link layer and SINR constraints at the physical layer in order to generate feasible matchings. We also presented a solution procedure based on column generation and provided numerical results that show the benefits of jointly controlling routing and scheduling.

\section{REFERENCES}

[1] P. Bjorklund, P. Varbrand and Di Yuan "Resource optimization of spatial TDMA in ad hoc radio networks: A column generation approach," In Proc. IEEE INFOCOM, pp. 818-824, Mar 2003.

[2] S.A. Borbash and A. Ephremides. "Wireless Link Scheduling With Power Control and SINR Constraints," IEEE Trans. Information Theory, vol. 52, no.11, pp. 5106-5111, Nov. 2006.

[3] S. Kompella, J.E. Wieselthier and A. Ephremides. "Multihop Routing and Scheduling in Wireless Networks Subject to SINR Constraints," to appear, In Proc. IEEE CDC, Dec. 2007.

[4] R. Nelson and L. Kleinrock. "Spatial-TDMA: A collision-free multihop channel access control," IEEE Trans. Communications, vol. 33 pp. 934-944, Sep. 1985.

[5] R.L. Cruz and A.V. Santhanam. "Optimal routing, link scheduling and power control in multihop wireless networks," In Proc. IEEE INFOCOM, pp. 702-711, Mar. 2003.

[6] T. ElBatt and A. Ephremides. "Joint Scheduling and Power Control for wireless ad-hoc networks," In Proc. IEEE INFOCOM, vol. 2, pp. 23-27, June 2002.

[7] J. Gronkvist "Assignment methods for spatial reuse TDMA," In Proc. IEEE Annual Workshop on Mobile and Ad Hoc Networking and Computing (MobiHOC), pp. 119-124, 2000.

[8] P. Gupta and P.R. Kumar. "The capacity of wireless networks," IEEE Trans. Information Theory, vol. 33, no. 2, pp. 388-404, Mar. 2000.

[9] S. Ramanathan and E.L. Lloyd. "Scheduling algorithms for multihop radio networks" IEEE/ACM Trans. Networking, vol. 1, no. 2, pp. 166-177, Apr. 1993.

[10] M.S. Bazaraa, J.J. Jarvis and H.D. Sherali. Linear Programming and Network Flows. John Wiley \& Sons: New York, NY, third edition, 2005. 